and to nuclear fallout, but relationship between occupational exposure to IR and thyroid pathologies still remains unclear.

Methods We ran a cross-section study to investigate whether healthcare workers routinely exposed to low-level IR had a higher prevalence of thyroid diseases. After giving written consent, 285 exposed workers (168 more exposed or 'A category' and 117 less exposed or 'B category') and 599 non-exposed were enrolled during routine examination at the Occupational Health Unite, programmed to monitor their health status. All subjects worked in the same university hospital and lived in the same geographical area of mild iodine deficiency. They were interviewed to fill in an anamnestic questionnaire (concerning to lifestyles, weariness habits and thyroid diseases risk factors) and underwent a physical examination, serum determination of fT3, fT4 and TSH, US neck scan and fine needle aspiration biopsy. Statistical analysis was performed using adjusted prevalence ratio (adPR) with software STATA 14.

Results Prevalence of thyroid diseases resulted statistically higher in exposed workers compared to controls (37\% vs $30 \%$, adPR $1.63 ; 95 \% \mathrm{CI}: 1.34$ to 1.97$)$. In particular, thyroid nodularity prevalence in exposed group was about twice as higher than controls (28\% vs 14\%; adPR 2.67; 95\% CI: 2.03 to 3.50). Any statistical association was found between IR exposure and malignant thyroid neoplasms (adPR 0.74; IC95\% 0.18-3.02). At multivariate analysis, female gender, age and familial history of thyroid diseases were significant risk factors.

Discussion In our study, mildly IR exposed health workers had a statistically higher thyroid diseases prevalence than control group, even though no difference was found between $\mathrm{A}$ and B categories. Results likely are due to closer and more meticulous health surveillance program carried out in IR exposed workers, allowing to identify thyroid alterations earlier than non-exposed health staf.

\section{STAFF COUNSELLING AND CONSULTATION IN AN ACUTE HOSPITAL SETTING. THE VALUE OF EVIDENCE BASED PRACTICE}

Pauline King *. Barbara Lynch MIACP, MIAHIP, MSc in Mindfulness Based Interventions

\subsection{6/oemed-2018-ICOHabstracts.927}

Introduction The Staff Counselling Service (SCS) promotes positive mental health and motivation by providing counselling, consultation, critical incident stress management (CISM) psycho-educational services, and Mindfulness Based Interventions (MBI's) to all staff in an acute hospital setting. An evaluation of the core counselling service commenced in 2016, to gather data on presenting issues and to ascertain the effectiveness of the service.

Methods Contextual statistical data such as client occupation, presenting issue, work related presentations (per Health Services Executive Management standards) are recorded and treatment outcomes are tracked via a web based Feedback Informed Treatment (FIT) system. Clients complete an Outcome Ratings Scale (ORS)1 at the beginning of each session to indicate their perception of how they are functioning and complete a Session Ratings Scale (SRS)2 at the end of each session scoring their experience of the session.

1 2000, Scott D. Miller and Barry L. Duncan

2 2000, Scott D. Miller and Barry L. Duncan
Results In 2016, 21\% of presenting cases were 'Work Related', the remaining 79\% were 'Personal' or 'Both' (work and personal). A breakdown of Work Related issues show 33\% due to Relationship issues; 21\% Support issues; 19\% 'CISM'; 16\% 'Demands' with the remaining 11\% relating to Role, Investigations and Work Injury. The average intake scores indicate that presentations were in line with scores typical for Out Patient Mental Health Settings. Client progress for those still in counselling were 2 times better than no treatment. For those who had completed counselling, progress was 5 times more than no treatment and above average compared to International clients with identical intake scores.

Discussion Data suggests that this is an effective counselling service. From a clinical perspective FIT facilitates ongoing collaboration and case review with clients. Low ORS scores can prompt timely onward referral to Occupational Health Physicians, allowing for clear dialogue, referencing measures which are easily understood. Work related presentations appear to support the ongoing provision of SCS interventions as a means of creating greater emotional intelligence and improving relationships.

\section{ASSESSING AND MANAGING JOB STRESS IN EMERGENCY DEPARTMENTS: WHICH TARGETS FOR IMPROVEMENT INTERVENTIONS?}

G d'Ettorre', V Pellicani'. 'Local Health Authority of Brindisi (ASL BR), Department of Occupational Medicine, Italy, ${ }^{2}$ Local Health Authority of Lecce (ASL LE), Department of Mental Health, Italy

\subsection{6/oemed-2018-ICOHabstracts.928}

Introduction The changes in the organisation of the healthcare system, actually involving the Italian healthcare hospital departments, triggered by the current economic crisis, in addition to ongoing technological innovation and developments in society, increase the exposure of healthcare workers (HCWs) to work related stress (WRS). Emergency Department (ED) workers are particularly vulnerable to WRS as a consequence of specific occupational risk factors typical of ED's healthcare professions.

Methods The authors conducted an integrated analysis of stress sentinel indicators and of objective stress factors of occupational context and content areas, among nurses and physicians of three Italian hospital EDs, in accordance to the multidimensional validated tool developed in Italy by National Network for Prevention of Work-Related Psychosocial Disorders. The aim of this observational study was to analyse the level of WRS after improvement interventions implemented by the management staff of EDs which focused on work context factors.

Results The assessment of WRS showed that nurses and physicians of the investigated EDs were exposed to a medium level of risk; the improvement interventions aimed at reducing WRS and focused on the following work context factors:

- Function and organisational culture;

- Role within the occupational organisation;

- Relationship at work, resulted significantly effective in minimising the risk of WRS.

Discussion In this study the implementation of improvement interventions focused on team development, safety training programs and adoption of the ethics code for healthcare 